\title{
Analysis of Dissolution of Salicylamide from Carrageenan Based Hard-Shell Capsules: A Study of the Drug-Matrix Interaction
}

\author{
Muhammad Al Rizqi Dharma Fauzi ${ }^{1}$, Esti Hendradii,*, Pratiwi Pudjiastuti ${ }^{1}$, and Riyanto Teguh \\ Widodo $^{3}$ \\ ${ }^{1}$ Department of Chemistry, Faculty of Science, Universitas Airlangga, Campus C Mulyorejo, Surabaya 60115, Indonesia \\ ${ }^{2}$ Department of Pharmaceutics, Faculty of Pharmacy, Universitas Airlangga, Campus C Mulyorejo, Surabaya 60115, Indonesia \\ ${ }^{3}$ Department of Pharmaceutical Technology, Faculty of Pharmacy, University of Malaya, Kuala Lumpur 50603, Malaysia
}

\section{* Corresponding author: \\ email:esti-h@ff.unair.ac.id}

Received: March 25, 2020

Accepted: September 22, 2020

DOI: $10.22146 / \mathrm{ijc} .55047$

\begin{abstract}
In drug release kinetics, the drug-matrix interaction is one of the important mechanisms to be dictated. Unfortunately, there is still minimum information discussing the effect of interaction between a drug and its matrix to the release profile of the drug. Therefore, there is an urgent need to conduct research related to the study of drug-matrix interaction. This paper reports the preparation of a drug delivery system (DDS) in the form of hard-shell capsules containing salicylamide (SCA) and analyses its drug-matrix interaction via dissolution test at different $\mathrm{pH}$ media and various release kinetics models. The matrix of hard-shell capsules was prepared from $\kappa$-carrageenan (CRG), crosslinked with maltodextrin (MD), and plasticized by sorbitol (SOR). The chemical properties of SCA were compared with paracetamol (PCT) using computational analysis to help to depict its drug-matrix interaction. The statistical analyses showed that SCA and PCT at $p H 1.2,4.5$, and 6.8 had all different release profiles. Based on the goodness of fit evaluation, the diffusion mechanism of SCA at pH 1.2 and 4.5 could be best described by the Peppas-Sahlin model while the zeroth-order model fitted the dissolution profile at $p H$ 6.8. In summary, it was proven that a different drug-matrix interaction produced a different dissolution profile.
\end{abstract}

Keywords: polymer; carrageenan; drug release; release kinetic; dissolution

\section{- INTRODUCTION}

Drug-matrix interaction is one of the most important mechanisms dictating the drug release profiles [1]. Unfortunately, there is minimum information discussing the effect of interaction between a drug and its matrix to its release profile, specifically for the matrix in the form of a hard-shell capsule. Press et al. [2] reported that polyether-based triblock tricopolymer micelles act as an agent to give the effect to the physicochemical properties of the matrix as well as the actual conditions during encapsulation interfere during the formulation of nanoparticular drug-matrix systems. On the other hand, drug molecules may directly interact with matrix molecules, lowering their solubility and/or retarding their release from the matrixes $[1,3]$. In addition, Srinarong et al. [3] stated that a fast drug dissolution with a high drug load such as diazepam could be obtained with the matrix such as polyvinylpyrrolidone that showed interaction with the drug. Therefore, there is an urgent need to study and conduct more research on drug-matrix interaction.

A matrix for a drug, which is also known as a drug delivery system (DDS), could be produced in different forms from different sources [4-5] where one of the common matrixes used in capsules [6]. Capsules used to be prepared from gelatin in the form of soft- or hardshell capsules [7]. As an inert material, capsules are a great DDS providing many advantages as compared to other DDS such as tablets and syrups [8]. Moreover, capsules can deliver both solid and liquid forms of drugs [9]. 
Fauzi et al. [6] prepared hard-shell capsules from CRG that were crosslinked with MD and plasticized by SOR. One pivotal advantage of this hard-shell capsule was that it had a longer disintegration time so that the dissolution process was easier to be observed as compared to hard gelatin capsules. It was found that PCT was more quickly dissolved at $\mathrm{pH} 4.5$ than that at $\mathrm{pH} 1.2$ and 6.8 due to the intermolecular interaction between PCT and citrate buffer in $\mathrm{pH} 4.5$ medium. However, this paper did not take into account the possibility of drug-matrix interactions that might contribute to this phenomenon.

Taking the aforementioned background into account, this paper reports the dissolution analysis of SCA released from CRG-MD/SOR capsules. In order to support the explanations about drug-matrix interaction, computational analysis was conducted to analyze the quantitative structure-activity relationship (QSAR) properties of SCA. Statistical study was evaluated using the DDSolver and Origin Pro 9 software to determine the significance of dissolution profiles of SCA and to determine the best model that could describe the release profiles. The goodness of fit data was used to describe the release mechanism of SCA from CRG-MD/SOR capsules at each acidity.

\section{- EXPERIMENTAL SECTION}

\section{Materials}

Food grade CRG powder was purchased from Kappa Carrageenan Nusantara, Inc., Pasuruan, Indonesia. Food grade MD powder was purchased from Yishui Dadi Corn Developing, Inc., Shandong, China. Pro analysis SOR was purchased from Sigma-Aldrich. Food grade lecithin was purchased from Tianjin Hexiyuan Lecithin Technology Inc., Pro analysis (purity: 99.0\%) while SCA was purchased from Sigma-Aldrich. Technical grade $\mathrm{HCl}$, citrate buffer, and phosphate buffer were all purchased from Sigma-Aldrich.

\section{Instrumentation}

Standard laboratory apparatuses such as beaker glass, volume pipette, hot plate, and others were used. Special apparatus for capsule preparation that was used is the size 0 dipping pens consisting of the body and head parts of hard-shell capsules. Several instruments were used such as Mettler Toledo NewClassic MS Balance to measure the mass of each material, Mettler Toledo SevenEasy to measure the $\mathrm{pH}$ value, Veego Dissolution Tester Type Dis 8000 to analyze the disintegration time of the capsules, and UV-Vis Spectrophotometer Shimadzu Type UV-1601 to obtain the absorbation of drug dissolved in the medium of dissolution analysis.

\section{Procedure}

\section{Capsules preparation}

CRG and MD were mixed in a ratio of mass (6:1 $\mathrm{w} / \mathrm{w})$. The mixture was diluted with $100 \mathrm{~mL}$ of deionized water, and then added with $1.5 \% \mathrm{v} / \mathrm{v}$ of SOR. This heterogeneous mixture was heated for $5 \mathrm{~h}$ until it formed a homogeneous mixture. Dipping pens used to print the capsules were dipped into this mixture and dried for $3 \mathrm{~h}$ at room temperature. Dried capsules were collected from dipping pens and cut neatly to produce a good shape of hard-shell capsules [6].

\section{Computational analysis for SCA and PCT}

The chemical properties of SCA and PCT were computed using HyperChem 8.0. Both geometries of molecules were optimized with the magnetic field of the Molecular Mechanics (MM+) field and with the conjugate gradient algorithm of Polak-Ribiere. After optimization, chemical properties were computed using the QSAR analysis method. The data analyzed was saved in hardware with the specifications of DELL type P24T with the processor of AMD A9-9420e RADEON R5 and with the core processor of 5 compute $2 \mathrm{C}+3 \mathrm{G} 1.80 \mathrm{GHz}$.

\section{In vitro dissolution medium preparation}

Three different media were prepared in this research to follow USP-711 (United States Pharmacopeia). A medium with standard $\mathrm{pH}$ of 1.2 to represent gastric acid fluid was obtained by diluting $52 \mathrm{~mL}$ of $\mathrm{HCl}(36.5 \%)$ in $10 \mathrm{~L}$ of deionized water, while a Standard $\mathrm{pH}$ of 4.5 to represent body fluid was obtained by dissolving $1.212 \mathrm{~g}$ of citric acid and $29.41 \mathrm{~g}$ of trisodium citrate dihydrate in $10 \mathrm{~L}$ of deionized water. Finally, a standard $\mathrm{pH}$ of 6.8 to represent intestine fluid was obtained by dissolving $2.844 \mathrm{mg}$ of $\mathrm{KH}_{2} \mathrm{PO}_{4}$ and $17.42 \mathrm{~g}$ of $\mathrm{K}_{2} \mathrm{HPO}_{4}$ in $10 \mathrm{~L}$ of deionized water. 


\section{In vitro dissolution test and drug-matrix interaction analysis}

At first, a standard curve was prepared for determining the concentration of SCA in each result. A primary standard solution of each medium was prepared by diluting $10.0 \mathrm{mg}$ of SCA in $100 \mathrm{~mL}$ of medium to produce a $100 \mathrm{ppm}$ solution. Afterward, $100 \mathrm{ppm}$ of SCA was diluted again to obtain $1,10,20,40$, and $50 \mathrm{ppm}$ of solutions. Standard curves of SCA in pH 1.2, 4.5, and 6.8 medium were made by plotting the absorbance of SCA solutions at $236.6 \mathrm{~nm}$ vs the concentrations.

Dissolution testing was conducted as per the USP711 recommendation. Six CRG-MD/SOR capsules containing SCA were placed in $900 \mathrm{~mL}$ of dissolution medium at $37 \pm 0.5^{\circ} \mathrm{C}$. A sample of $10 \mathrm{~mL}$ solution from each of the vessels was taken out at a pre-determined time interval and replaced it by $10 \mathrm{~mL}$ of the fresh dissolution medium to maintain sink condition. The concentration of SCA in all samples collected was measured using UV-Vis Spectrophotometer at $236.6 \mathrm{~nm}$ [10]. Profile dissolutions were then built-up by plotting between accumulated concentrations against interval times.

\section{Release kinetic analysis}

Five candidates of release kinetics models were identified to fit the dissolution of SCA from CRGMD/SOR, namely zero order, first order, Higuchi, RitgerPeppas, and the Peppas-Sahlin model [4]. They can be expressed by Eq. (1) to (5), respectively.

$\mathrm{Q}_{\mathrm{f}}=\mathrm{k}_{0} \mathrm{t}+\mathrm{Q}_{0}$

where $\mathrm{Q}$ is the accumulated concentration, $\mathrm{k}_{0}$ is the zeroth-order release kinetics constant, and $\mathrm{t}$ is time.

$\ln \mathrm{Q}_{\mathrm{f}}=\mathrm{k}_{1} \mathrm{t}+\ln \mathrm{Q}_{0}$

where $\mathrm{k}_{1}$ is the first-order release kinetics constant.

$\mathrm{Q}=\mathrm{k}_{\mathrm{H}} \sqrt{\mathrm{t}}$

where $\mathrm{k}_{\mathrm{H}}$ is the Higuchi constant.

$\mathrm{Q}=\mathrm{k}_{\mathrm{p}} \mathrm{t}^{\mathrm{n}}$

where $k_{p}$ is the Ritger-Peppas constant and $n$ is diffusion exponential. Ritger-Peppas equation is also known as the Power Law. The value of $\mathrm{n}$ will give specific information about the diffusion mechanism experienced by the drug released from the matrix. For the cylinder-like matrix [11], if $\mathrm{n}=0.45$, the mechanism will be the Fickian
Diffusion. If the value of $\mathrm{n}$ is $0.45<\mathrm{n}<0.89$, the mechanism will be the Anomalous (Non-Fickian) Transport. The Case-II Transport mechanism does not occur for $\mathrm{n}=0.89$ due to geometric factors involved in the related mathematical analysis [12]. Therefore, $\mathrm{n}=$ 0.89 only indicates a non-Fickian transport. If $\mathrm{n}>0.89$, the mechanism will be the Super-Case-II Transport.

$\mathrm{Q}=\mathrm{k}_{\mathrm{D}} \mathrm{t}^{\mathrm{n}}+\mathrm{k}_{\mathrm{R}} \mathrm{t}^{\mathrm{n}}$

where $k_{D}$ is the diffusion constant and $k_{R}$ is the relaxational constant. Uliniuc et al. [13] explained that the interaction mechanism between drugs and matrixes will be changed if the swelling process happens. In addition, material that is able to be swollen tends to have a non-Fickian diffusion mechanism [4]. In order to determine the best model that fits the release profile, statistical analysis was used.

\section{Statistical analysis}

Statistical analysis using Origin Pro 9 and DDSolver software was employed to compare and evaluate the different concentrations of SCA dissolved/released at each point of the three dissolution profiles obtained. The best model that fits the drug release profile was determined by their goodness of fit. The parameters used for the goodness of fit were $R^{2}$, mean squared error (MSE), sums of squares (SS), and Akaike Information Criterion (AIC) [4,14]. AIC was used as a primary determination since most studies tend to use this method as a diagnostic criterion for model selection [14]. Eq. (6) shows the expression for AIC evaluation [15].

$\mathrm{AIC}=\mathrm{N}(\ln R S S)+2 \mathrm{p}$

where $\mathrm{N}$ is the number of experimental data points, $\mathrm{p}$ is the number of parameters in an estimated model, and RSS is the residual sum of squares.

\section{- RESULTS AND DISCUSSION}

\section{Computational Analysis of SCA and its Comparison with PCT}

To collect deeper information about the drugmatrix interaction, we compared SCA and PCT using computational analysis. This helped us to depict the result of the SCA interaction with the CRG-MD/SOR 
capsules. Both SCA and PCT contained phenol and amide groups. The difference is the hydroxyl groups of PCT and SCA on the para and meta to the amide groups respectively. It makes SCA narrower in surface area and smaller in volume than PCT. Table 1 shows the results of the computational analysis using HyperChem 8.0.

PCT and SCA as shown in Fig. 1 have a close polarizability value since they consist of the same groups. However, the difference between the amides that bond the benzene rings might be the ones that are affecting the polarizability. In PCT, the benzene ring directly binds with the atom $\mathrm{N}$ of the amide group that makes it act as an electron-donating group. On the contrary, the benzene ring in SCA binds the carbonyl of the amide to act as an electron-withdrawing group. Thus, electrons in SCA distributed more equal than that of PCT, making PCT a more polar compound than SCA.

It is known that the pKa of PCT is 9.38 [16] and the $\mathrm{pKa}$ of SCA is 8.20 [17]. One possible explanation is that the hydroxyl group of SCA that is close to the amide group might create an intramolecular hydrogen bond and thus reducing the ability of the amide group to donate its electron-pair. This circumstance might not be found in PCT and make it a better Lewis-base compound.

\section{Dissolution Profiles of SCA Released from CRG- MD/SOR Capsules}

SCA (Fig. 2) was dissolved well at $\mathrm{pH} 1.2$ and 4.5 but was poorly dissolved at $\mathrm{pH}$ 6.8. In this almost-basic medium, even in $90 \mathrm{~min}$, the SCA was dissolved for only (a)<smiles>CC(=O)Nc1ccc(O)cc1</smiles>

(b)<smiles>NC(=O)c1ccccc1O</smiles>

Fig 1. Optimized chemical structure of (a) paracetamol (PCT) and (b) salicylamide (SCA) using HyperChem 8.0

Table 1. Computational analysis results of paracetamol (PCT) and salicylamide (SCA) using HyperChem 8.0

\begin{tabular}{llcc}
\hline \multirow{2}{*}{ No } & \multirow{2}{*}{ QSAR Properties } & \multicolumn{2}{c}{ Compound } \\
\cline { 3 - 4 } & & PCT & \multicolumn{2}{c}{$\mathrm{SCA}$} \\
$\mathrm{C}_{8} \mathrm{H}_{9} \mathrm{NO}_{2}$ & $\mathrm{C}_{7} \mathrm{H}_{7} \mathrm{NO}_{2}$ \\
\hline 1 & Net charge & $0.00 \mathrm{e}$ & $0.00 \mathrm{e}$ \\
2 & Surface area (approx.) & $299.39 \AA^{2}$ & $216.45 \AA^{2}$ \\
3 & Volume & $488.76 \AA^{3}$ & $422.73 \AA^{3}$ \\
4 & Log P & -1.32 & -0.91 \\
5 & Refractivity & $45.55 \AA^{3}$ & $40.38 \AA^{3}$ \\
6 & Polarizability & $16.18 \AA^{3}$ & $14.34 \AA^{3}$ \\
7 & Molecular Mass (amu) & 151.16 & 137.14 \\
\hline
\end{tabular}

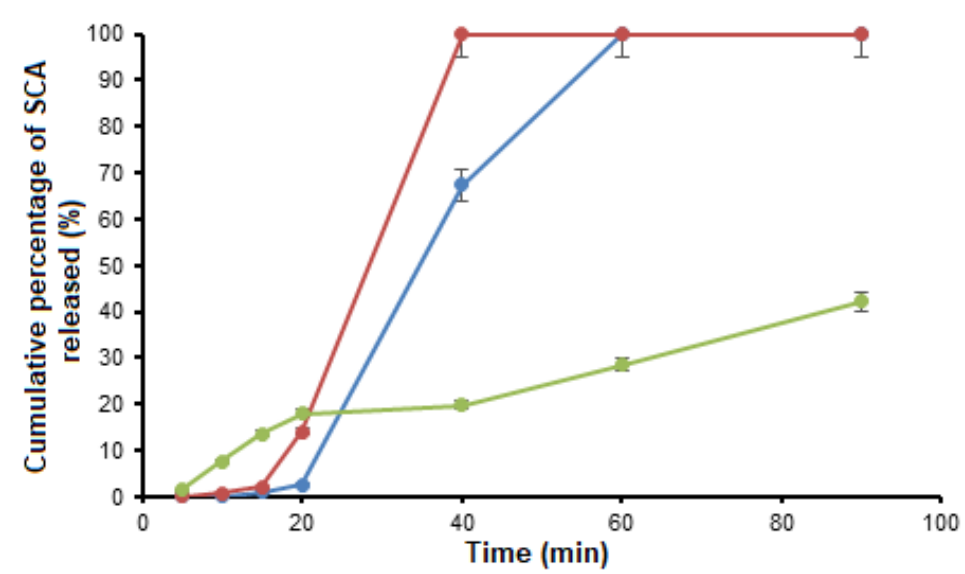

Fig 2. The dissolution profile of salicylamide (SCA) from carrageenan-maltodextrin plasticized by sorbitol (CRG$\mathrm{MD} / \mathrm{SOR}$ ) capsules at $\mathrm{pH} 1.2$ (blue), 4.5 (red), and 6.8 (green) 
$42.19 \pm 7.22 \%$. One possible explanation is that SCA is a weak-basic drug. The consequence of this fact supports the mentioned results that the drugs were dissolved poorly at $\mathrm{pH}$ 6.8. In addition, CRG-MD/SOR capsules were not recommended for drugs targeted at the intestinal parts [6].

Using the similarity factor $\left(\mathrm{f}_{2}\right)$ method on DDSolver, the difference between SCA and PCT [6] could be determined. This method is used because the dissolution plateau has been reached (> 85\%) [14]. The value of $\mathrm{f}_{2}$ at pH 1.2 was 39.40 which indicated no similarity between the two profiles. The same condition also happened to the profiles at $\mathrm{pH} 4.5$ where the value of $\mathrm{f}_{2}$ was 35.83 . The profiles at $\mathrm{pH} 6.8$ were analyzed using the multivariate confidence region method since both dissolution profiles did not reach $85 \%$. The advantage of the multivariate method is that it takes the variability and correlation structure of the data into account in measuring the difference between the means of two sets of dissolution data [14]. The maximum difference means fraction used was $10.0 \%$ and the maximum multivariate statistical distance of the profiles was 28.32. This indicates that there was no similarity between SCA and PCT [6] at pH 6.8.

This difference might be caused by the acidity that affects the activity of the amide and phenol groups on SCA. At $\mathrm{pH} 1.2$, the amide groups might be protonated more than that of $\mathrm{pH}$ 4.5. This protonated state increased the radius of the amide group so that the molecules might not interact well on the network of CRG-MD/SOR capsules due to the Van der Waals interaction that is affected by the size of the molecules [18]. On the other side, SCA would interact better with each other. When the medium penetrated the matrix, the less interaction between SCA and CRG-MD/SOR resulted in a slow dissolution process of SCA compared to that of $\mathrm{pH} 4.5$ because the medium would take a longer time to interact with SCA that interacted better with each other.

\section{In Vitro Release Kinetics Analysis of SCA}

All release kinetics models are valid at $60 \%$ of initial drug release from the capsules [19]. Hence, all of the calculations to determine each coefficient and $\mathrm{R}^{2}$ were obtained from the $60 \%$ data of drug released. This validity was pivotal, especially in the determination of the AIC values for each release kinetics model. The values depended on the number of data involved in the calculation. Based on the lowest AIC value, as shown in Table 2, the Peppas-Sahlin model was the best model to describe the release kinetics mechanism of SCA at $\mathrm{pH}$ 1.2. This suggestion was supported by high $\mathrm{R}^{2}$ and low MSE and SS, where the lowest value indicated the data had low deviancy [4].

The zeroth-order model could not be applied to the release kinetics of SCA, because an ideal carrier for zeroth-order release should have a constant release rate over the entire release duration [20]. First-order model also could not be applied because the carrier must be a non-swelling porous matrix [21]. The Higuchi model in this report had the highest AIC value, meaning it really could not describe the release process. It is because the

Table 2. Statistical results of salicylamide (SCA) released from carrageenan-maltodextrin plasticized by sorbitol (CRG$\mathrm{MD} / \mathrm{SOR}$ ) capsules at pH 1.2. DDSolver was used to analyze zeroth-order, first-order, Higuchi model, and PeppasSahlin model. Since the software could not be able to analyze the Peppas-Ritger model, Origin Pro 9 was used instead

\begin{tabular}{llcccc}
\hline Model & Constants & $\mathrm{R}^{2}$ & MSE & SS & AIC \\
\hline Zeroth-Order & 1.17 & 0.63 & 321.29 & 1285.15 & 37.76 \\
First-Order & 0.01 & 0.55 & 395.63 & 1582.52 & 38.79 \\
Higuchi & 4.89 & 0.33 & 589.09 & 2356.36 & 40.79 \\
Peppas-Ritger & & & & & \\
$\mathrm{n}$ & 4.43 & 0.99 & 0.51 & 1622.42 & 40.96 \\
$\mathrm{k}_{\mathrm{p}}$ & $5.18 \times 10^{-6}$ & & & & \\
Peppas-Sahlin & & & & & \\
$\mathrm{k}_{\mathrm{D}}$ & $5.00 \times 10^{-6}$ & 0.99 & 0.42 & & \\
$\mathrm{k}_{\mathrm{R}}$ & $4.00 \times 10^{-13}$ & & & & \\
\hline
\end{tabular}


Higuchi model is expected to happen in a drug that is dispersed in a uniform matrix [22]. Based on the PeppasRitger model, the exponential constant (n) was 4.43 . According to Paarakh et al. [23], if $\mathrm{n}$ is higher than 0.85, the diffusion mechanism will be the Super-Case-II transport. This might be caused by the capsules that were swollen for more than 15 min before finally disintegrated and dissolved. The swelling of the capsule might be the primary reason for non-Fickian diffusion that happened to CRG-MD/SOR. The Peppas-Sahlin model fitted the release profiles at $\mathrm{pH} 1.2$ and 4.5 (Table 3) according to AIC evaluation. Small diffusion and relaxation constants at both acidities also showed that the diffusion took a longer time.

The predicted equations formed by the parameters that were calculated by DDSolver in the Peppas-Sahlin model were processed by the program to form the predicted graphs (Fig. 3). When all observed points were fitted to the graphs, they demonstrated good performances. Fig. 3 showed that the Peppas-Sahlin model could describe the release kinetics of SCA from CRG-MD/SOR capsules at $\mathrm{pH} 1.2$ and 4.5.

Eq. (5) can be rewritten as:

$\mathrm{Q}=\mathrm{k}_{\mathrm{D}} \mathrm{t}^{\mathrm{n}}+\left(1+\frac{\mathrm{k}_{\mathrm{R}}}{\mathrm{k}_{\mathrm{D}}} \mathrm{t}^{\mathrm{n}}\right)$

Based on Eq. (7), the percentage of drug release due to the Fickian mechanism (F) is clearly calculated as [11]:

$\frac{1}{\mathrm{~F}}=\frac{\mathrm{k}_{2}}{\mathrm{k}_{1}} \mathrm{t}^{\mathrm{n}}+1$

By plotting $1 / \mathrm{F}$ as $\mathrm{x}$-axis and $\mathrm{t}^{\mathrm{n}}$ as $\mathrm{y}$-axis, Fig. 4 was produced.

Fig. 3 shows the diffusion of SCA more rapidly transforms from Fickian to non-Fickian diffusion at $\mathrm{pH}$ 4.5 than that of $\mathrm{pH}$ 1.2. This was possibly due to the different relaxation constant of CRG-MD/SOR at $\mathrm{pH} 4.5$

Table 3. Statistical results of salicylamide (SCA) released from carrageenan-maltodextrin plasticized by sorbitol (CRG$\mathrm{MD} / \mathrm{SOR}$ ) capsules at $\mathrm{pH}$ 4.5. DDSolver was used to analyze zeroth-order, first-order, Higuchi model, and PeppasSahlin model. Since the software could not be able to analyze the Peppas-Ritger model, Origin Pro 9 was used instead

\begin{tabular}{lllrcc}
\hline Model & Constants & \multicolumn{1}{c}{$\mathrm{R}^{2}$} & \multicolumn{1}{c}{ MSE } & \multicolumn{1}{c}{ SS } & \multicolumn{1}{c}{ AIC } \\
\hline Zeroth-Order & 1.84 & 0.68 & 610.90 & 2443.60 & 40.91 \\
First-Order & 0.02 & 0.54 & 875.46 & 3501.84 & 42.77 \\
Higuchi & 7.84 & 0.47 & 1210.53 & 4842.11 & 44.41 \\
Peppas-Ritger & & & & & \\
$\mathrm{n}$ & 4.25 & 0.94 & 1.83 & 1487.21 & 33.22 \\
$\mathrm{k}_{\mathrm{p}}$ & $3.25 \times 10^{-5}$ & & & & \\
Peppas-Sahlin & & & & & \\
$\mathrm{k}_{\mathrm{D}}$ & $4.00 \times 10^{-5}$ & 0.99 & 39.65 & 79.31 & 19.50 \\
$\mathrm{k}_{\mathrm{R}}$ & $1.00 \times 10^{-10}$ & & & & \\
\hline
\end{tabular}
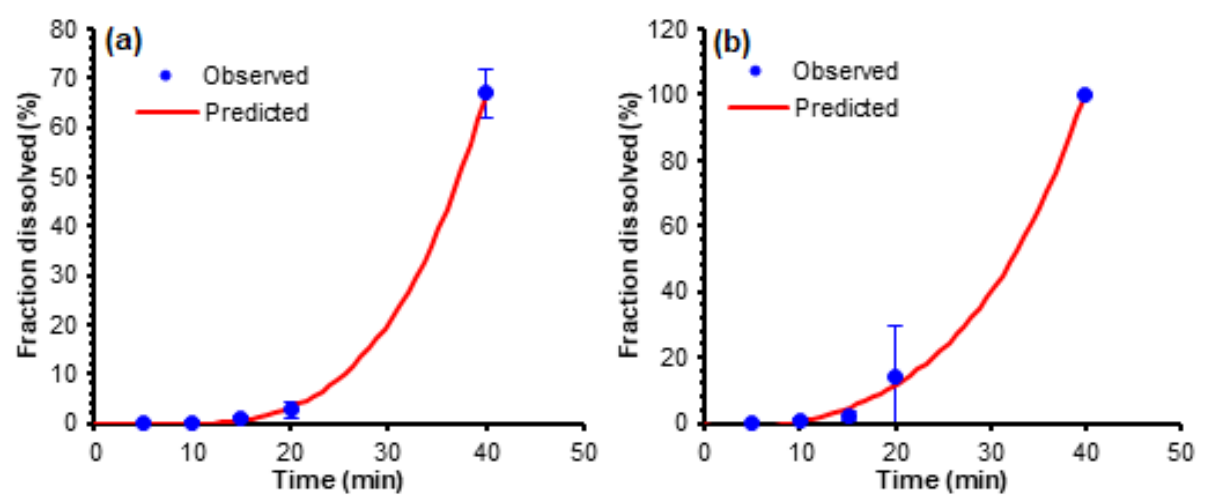

Fig 3. Predicted graphs of the Peppas-Sahlin model of salicylamide (SCA) release kinetics at (a) pH 1.2 and (b) pH 4.5. DDSolver was used to analyze the performance of the model 


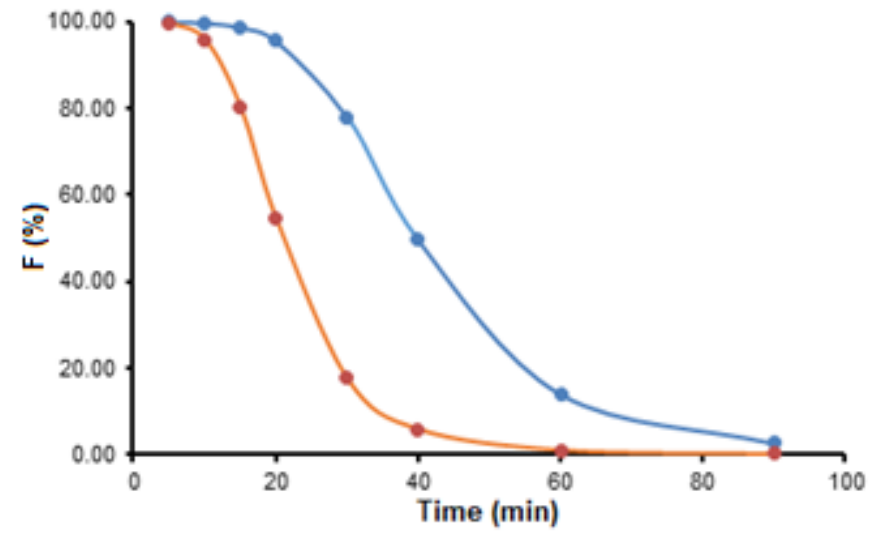

Fig 4. Fickian diffusion percentage (F) of salicylamide (SCA) vs time at $\mathrm{pH} 1.2$ (blue) and 4.5 (red)

and 1.2 where the former showed 200 higher than the latter. As a consequence, CRG-MD/SOR at $\mathrm{pH} 4.5$ exhibited faster swelling and transition from glassy to rubbery state [24]. Hence, faster swelling would cause faster disintegration and dissolution.

Based on our analysis, DDSolver could not be used accurately for a dissolution profile that did not reach $60 \%$. Based on AIC value where it was 33.06 for the PeppasSahlin model and 32.69 for the first-order model, the release mechanism of SCA from CRG-MD/SOR capsules was best described by the first-order model. Interestingly, the MSE and SS of the first-order model were 13.07 and 65.34. These parameters were higher than those of the Peppas-Sahlin model, which the MSE and SS of it were 12.82 and 51.28 , respectively. These results indicate that the first-order model had high deviancy compared to that of the Peppas-Sahlin model. The results were contradictory. Therefore, we chose the results from Origin Pro 9 calculation to analyze the release mechanism of SCA from CRG-MD/SOR capsules at $\mathrm{pH} 6.8$.

Based on Table 4, the best model to describe the release kinetic process at $\mathrm{pH} 6.8$ was the zeroth-order model. The diffusion follows the zeroth-order, indicating that the capsule acted as a nonswelling porous matrix [21]. This also indicated that Fickian diffusion was the dominant mechanism occurring in that condition. This conclusion was supported by the fact that the release process at $\mathrm{pH} 6.8$ was slow where only $42.19 \pm 7.22 \%$ of SCA was released in $90 \mathrm{~min}$. The analysis was supported by Fig. 5 where the observed data fitted well on the zeroth-order model.

Diffusion and relaxation constant could also be interpreted as a competition between Fickian and nonFickian diffusion [4]. By modifying Eq. (7), the ratio of relaxational over Fickian contribution can be expressed by Eq. (9) as follows:

$\frac{\mathrm{R}}{\mathrm{F}}=\frac{\mathrm{k}_{2}}{\mathrm{k}_{1}} \mathrm{t}^{\mathrm{n}}$

The increase in $\mathrm{R} / \mathrm{F}$ indicated the increase of relaxation contribution and thus increasing the nonFickian diffusion percentage [4]. Fig. 6 indicated that the non-Fickian diffusion was dominant at $\mathrm{pH} 1.2$ and 4.5 while the Fickian diffusion was dominant at $\mathrm{pH} 6.8$. Therefore, the dissolution of SCA from CRG-MD/SOR capsules depended on the acidity of the medium. The determination of the diffusion mechanism was helpful to understand the dissolution process.

Table 4. Statistical results of salicylamide (SCA) released from carrageenan-maltodextrin plasticized by sorbitol (CRG$\mathrm{MD} / \mathrm{SOR}$ ) capsules at $\mathrm{pH}$ 6.8. The analyze was done using Origin Pro 9

\begin{tabular}{lcccc}
\hline Model & Constant & $\mathrm{R}^{2}$ & \multicolumn{1}{c}{ RSS } & AIC \\
\hline Zeroth-Order & 0.42 & 0.94 & 63.50 & 33.06 \\
First-Order & 0.03 & 0.61 & 2738.17 & 59.40 \\
Higuchi & 5.02 & 0.96 & 1954.54 & 57.04 \\
Peppas-Ritger & & & & \\
$\mathrm{n}$ & 1.09 & 0.82 & 106.42 & 38.67 \\
$\mathrm{k}_{\mathrm{p}}$ & 0.41 & & & \\
Peppas-Sahlin & & & & \\
$\mathrm{k}_{\mathrm{D}}$ & 0.27 & 0.85 & 5717.10 & 68.56 \\
$\mathrm{k}_{\mathrm{R}}$ & 0.0018 & & & \\
\hline
\end{tabular}




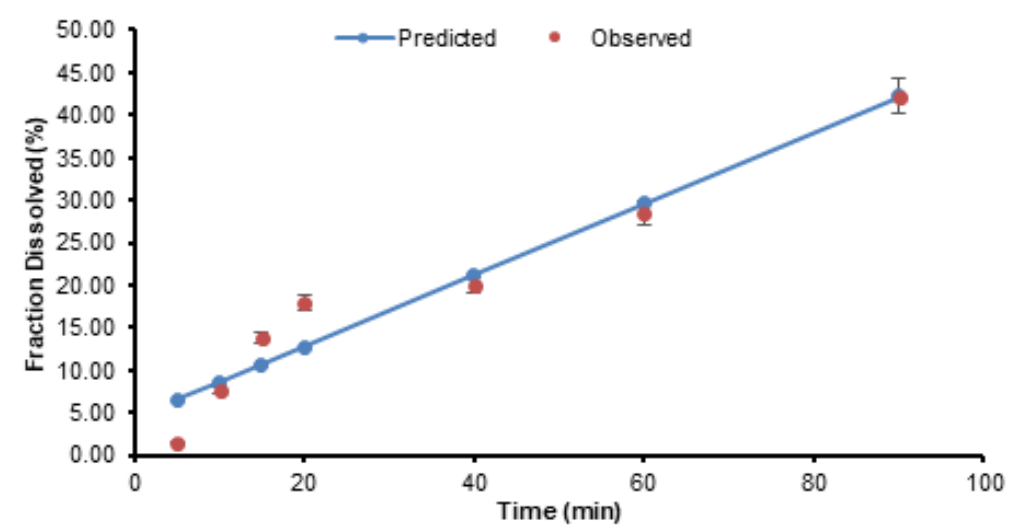

Fig 5. Predicted graphs of the zeroth-order model of salicylamide (SCA) release kinetics at pH 6.8

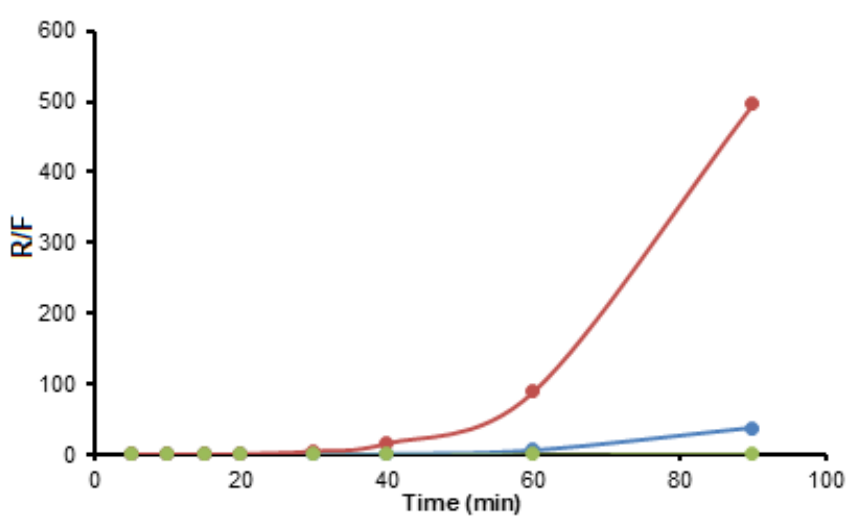

Fig 6. Relaxation process and its Fickian contribution ratio of salicylamide (SCA) dissolution at $\mathrm{pH} 1.2$ (blue), pH 4.5 (red), and pH 6.8 (green)

\section{- CONCLUSION}

Based on the computational analyses, SCA and PCT have significantly different drug-matrix interactions due to their different functional groups' position where the significance was clearly seen at $\mathrm{pH} 1.2$ and 4.5. The benzene ring in SCA binds the carbonyl of the amide that makes it act as an electron withdrawing group, making SCA easier to be protonated compared to PCT. This fact supports the fact that SCA and PCT have different results in their interaction with the CRG-MD/SOR capsules, especially at $\mathrm{pH} 1.2$ and 4.5 where SCA was released faster at $\mathrm{pH} 4.5$ than at $\mathrm{pH} 1.2$. In other words, the drug-matrix interaction affected the ability of the medium to dissolve the SCA. Finally, the diffusion mechanism of SCA at pH 1.2 and 4.5 could be best described by the Peppas-Sahlin model while the first-order model fitted the dissolution profile at $\mathrm{pH} 6.8$.

\section{- ACKNOWLEDGMENTS}

The authors would like to thank the Ministry of Education of Indonesia for the research fund granted to us through the PMDSU (Program Menuju Doktor untuk Sarjana Unggul) scholarship program.

\section{- AUTHOR CONTRIBUTIONS}

Muhammad Al Rizqi Dharma Fauzi conducted the research and analyses, Esti Hendradi supervised the research conducted, Pratiwi Pudjiastuti and Riyanto Teguh Widodo reviewed the manuscript written. All authors agreed to the final version of this manuscript.

\section{- REFERENCES}

[1] Zeng, L., An, L., and Wu, X., 2011, Modelling drugcarrier interaction in the drug release from nanocarriers, J. Drug Delivery, 2011, 370308.

[2] Press, A.T., Ramoji, A., von der Lühe, M., Rinkenauer, A.C., Hoff, J., Butans, M., Rössel, C., Pietsch, C., Neugebauer, U., Schacher, F.H., and Bauer, M., 2017, Cargo-carrier interactions significantly contribute to micellar conformation and biodistribution, NPG Asia Mater., 9, e444.

[3] Srinarong, P., Kouwen, S., Visser, M.R., Hinrichs, W.L.J., and Frijlink, H.W., 2010, Effect of drugcarrier interaction on the dissolution behavior of solid dispersion tablets, Pharm. Dev. Technol., 15 (5), 460-468.

[4] Unagolla, J.M., and Jayasuriya, A.C., 2018, Drug transport mechanisms, and in vitro release kinetics of vancomycin encapsulated chitosan-alginate 
polyelectrolyte microparticles as a controlled drug delivery system, Eur. J. Pharm. Sci., 114, 199-209.

[5] Hariyadi, D.M., Hendradi, E., and Sharon, N., 2019, Development of carrageenan polymer for encapsulation of ciprofloxacin HCL: In vitro characterization, Int. J. Drug Delivery Technol., 9 (1), 89-93.

[6] Fauzi, M.A.R.D., Pudjiastuti, P., Hendradi, E., Widodo, R.T., and Amin, M.C.I.M., 2020, Characterization, disintegration, and dissolution analyses of carrageenan based hard-shell capsules cross-linked with maltodextrin as a potential alternative drug delivery system, Int. J. Polym. Sci., 2020, 3565931.

[7] The United States Pharmacopoeial Convention, 2014, United States Pharmacopoeia (USP), Rockville, Maryland.

[8] Srividya, B., Sowmya, C., and Chappidi, S.R., 2014, Capsules and its technology: An overview, Int. J. Pharm. Drug Anal., 2 (9), 727-733

[9] Gullapalli, R.P., and Mazzitelli, C.L., 2017, Gelatin and non-gelatin capsule dosage forms, J. Pharm. Sci., 106 (6), 1453-1465.

[10] Ali, N.W., Zaazaa, H.E., and Abdelrahman, M.M., 2014, Novel spectrophotometric methods for determination of salicylamide and ascorbic acid in their binary mixture, J. Chem. Soc. Pak., 36 (6), 988995.

[11] Peppas, N.A., and Sahlin, J.J., 1989, A simple equation for the description of solute release. III. Coupling of diffusion and relaxation, Int. J. Pharm., 57 (2), 169-172.

[12] Peppas, N.A., 1985, Analysis of Fickian and nonFickian drug release from polymers, Pharm. Acta Helv., 60 (4), 110-111

[13] Uliniuc, A., Hamaide, T., Popa, M., and Băcăiţă, S., 2014, Modified starch-based hydrogels cross-linked with citric acid and their use as drug delivery systems for levofloxacin, Soft Mater., 11 (4), 483-493.

[14] Zhang, Y., Huo, M., Zhou, J., Zou, A., Li, W., Yao, C., and Xie, S., 2010, DDSolver: An add-in program for modeling and comparison of drug dissolution profiles, AAPS J., 12 (3), 263-271.

[15] Kulpreechanan, N., and Sorasitthiyanukarn, F.N., 2020, Evaluation of in vitro release kinetics of Capsaicin-loaded chitosan nanoparticles using DDSolver, Int. J. Res. Pharm. Sci., 11 (3), 45554559.

[16] Bernal, V., Erto, A., Giraldo, L., and Piraján, J.C.M., 2017, Effect of solution $\mathrm{pH}$ on the adsorption of paracetamol on chemistry modified activated carbons, Molecules, 22 (7), 1032.

[17] Lemke, T.L., Williams, D.A., Roche, V.F., and Zito, S.W., 2017, Foye's Principles of Medicinal Chemistry, $7^{\text {th }}$ Ed., Lippincott Williams \& Wilkins, Philadelphia, USA, 965-970.

[18] Hermann, J., DiStasio, R.A., and Tkachenko, A., 2017, First-principles models for van der Waals interactions in molecules and materials: Concepts, theory, and applications, Chem. Rev., 117 (6), 47144758.

[19] Fu, T., and Kao, W.J., 2010, Drug release kinetics and transport mechanisms of non-degradable and degradable polymeric delivery systems, Expert Opin. Drug Delivery, 7 (4), 429-444.

[20] Zhao, Y.N., Xu, X., Wen, N., Song, R., Meng, Q., Guan, Y., Cheng, S., Cao, D., Dong, Y., Qie, J., Liu, K., and Zhang, K., 2017, A drug carrier for sustained zero order release of peptide therapeutics, Sci. Rep., 7, 5524.

[21] Varelas, C.G., Dixon, D.G., and Steiner, C.A., 1995, Zero-order release from biphasic polymer hydrogels, J. Controlled Release, 34 (3), 185-192.

[22] Paul, D.R., 2011, Elaborations on the Higuchi model for drug delivery, Int. J. Pharm., 418 (1), 13-17.

[23] Paarakh, M.P., Jose, P.A., Setty, C.M., and Christoper, G.V.P., 2018, Release kinetics Concepts and applications, IJPRT., 8, 12-20.

[24] Diksha, S., Dhruv, D., Prasad D.N., and Mansi, H., 2019, Sustained release drug delivery system with the role of natural polymers: A review, J. Drug Delivery Ther., 9 (3-s), 913-923. 